\title{
SD Rat Liver Polydatin Concentration Control Based On Sliding Mode with High-gain Observer
}

\author{
Jian-Yang LIN ${ }^{1,}$, Yu-Shuai BU², Jin-Rui GAO ${ }^{2}$ \\ ${ }^{1}$ Department of Pharmacy, the First Hospital of China Medical University, \\ Shenyang City, China \\ ${ }^{2}$ School of Automobile and Transportation, Shenyang Ligong University, \\ Shenyang City, China \\ E-mail address: linjianyangcmu@126.com \\ ${ }^{*}$ Corresponding author
}

Keywords: Polydatin, SD rats, Liver tissues, Sliding mode control.

\begin{abstract}
In this paper, we use the sliding mode control based on high gain observer to control the concentration of Polydatin in the liver of SD rates. Through data processing and with the help of the nlinfit function in MATLAB, we take a fitting analysis by using the Polydatin distribution data in SD rats' liver tissues and achieve a very good fitting effect. Based on the fitting function that have got, we take the sliding mode control to design observer and controller for controlling the output and creating MATLAB / Simulink simulation program. The mathematical model that we made is clear and accurate, from which, we can easily observe and analyze the input-output curves, estimate effectively the results, achieve our purpose that control the output by controlling the input. Simulation results show that the control algorithm have certain robustness and can achieve a good result to the external disturbance and parameter uncertainty.
\end{abstract}

\section{Introduction}

Modern pharmacological studies show that the Polydatin can improve microcirculation, inhibit the release of lysosomal enzymes, lower blood pressure, anti-lipid peroxidation, anti-platelet aggregation, anti-platelet aggregation, cure cough and asthma, anti-bacterial, anti-viral and so on [1-3]. The polydatin injections can be used to treat myocardial ischemia, cerebral ischemia, shock and other cardiovascular cerebrovascular diseases, to reduce tissue and organ damages caused by a variety of factors $[4,5]$. Taking effect in cells, Polydatin provides a theoretical guidance for developing new drugs and studying shock mechanism [6, 7]. Sliding mode control is a special control strategy, with a simple algorithm, have a advantage of insensitivity to parameter changes and disturbances, no system online identification, physical implementation simplicity, robustness and high reliability [8], it is widely used in a various of control systems in engineering practice, especially for the deterministic control system that can establish a accurate mathematical model. This control method makes the system state slide along the sliding surface by switching the control parameters, it also makes the system uncertain when it is affected by the proactive move parameters and external interference. It is the feature that makes the sliding mode control method attention of scholars $[9,10]$. 


\section{Data [7]}

SD rats were intravenously injected with a single dose administration of $20 \mathrm{mg} / \mathrm{Kg}$ Polydatin, the data of drug distribution in liver tissue are as following Table1.

Table 1. The data of drug distribution in liver tissue

\begin{tabular}{|c|c|}
\hline Time $(\mathrm{min})$ & Liver tissue drug concentration $(\mu / g))$ \\
\hline 10 & $1.74 \pm 0.46$ \\
\hline 40 & $0.38 \pm 0.02$ \\
\hline 60 & $0.27 \pm 0.13$ \\
\hline 120 & $0.15 \pm 0.09$ \\
\hline 240 & $0.11 \pm 0.02$ \\
\hline
\end{tabular}

\section{Establishing Two-compartment Model [11, 12]}

\section{Establishing Pharmacokinetic Model}

The amount of drugs $x_{l}(t)$ in absorption chamber (such as blood) satisfies the following differential formula (1):

$$
\frac{d x_{1}(t)}{d t}=-k_{1} x_{1}(t), \quad x_{1}(0)=m c_{0}
$$

In formula (1), $k_{l}$ is the ratio of drug's rate(the amount of the drugs reduces in per unit time in the absorption chamber), from absorption chamber (or blood) to central chamber (such as liver), and the amount of drug in absorption chamber. $m c_{0}$ is the total amount of drugs, $m$ is the mass of SD rats, $c_{0}$ is the ratio.

The amount of drugs in Central chamber (such as liver) satisfies the differential formula (2):

$$
\frac{d y_{1}(t)}{d t}=k_{1} x_{1}(t)-k_{2} y_{1}(t), \quad y_{1}(0)=0
$$

In formula (2), $k_{2}$ is the ratio of drug's rate (the amount of drugs reduces in per unit time in the central chamber), from the central chamber to vitro, and the amount of drugs in the central compartment.

We solve the above formula (2) initial results by using MATLAB, the results are as follows formula (3).

$$
\left\{\begin{array}{l}
x_{1}(t)=m c_{0} e^{-k_{1} t} \\
y_{1}(t)=\frac{m c_{0} k_{1}}{k_{1}-k_{2}}\left(e^{-k_{2} t}-e^{-k_{1} t}\right)
\end{array}\right.
$$

Note $c(t)=y_{l}(t) / m_{1}$, among them, $m_{1}$ is the mass of the liver, bringing $c(t)$ into the formula (3), we can obtain as follows formula(4): 


$$
c(t)=k\left(e^{-k_{2} t}-e^{-k_{1} t}\right)
$$

In formula (4), $k=m c_{0} k_{1} / m_{1}\left(k_{1}-k_{2}\right), k_{1} \neq k_{2}$.

Formula (4) is the mathematical model of drug content in liver that be established after rapid intravenous injection of Polygonum,

\section{Fitting the Data}

We use the nlinfit (nonlinear least squares fit) function in MATLAB $[13,14]$ to fit the data in Table 1, the results are as follows formula(5):

$$
c(t)=2.7083\left(e^{-0.0447 t}-e^{-2.9674 t}\right)
$$

The fitting results are shown in Fig. 1

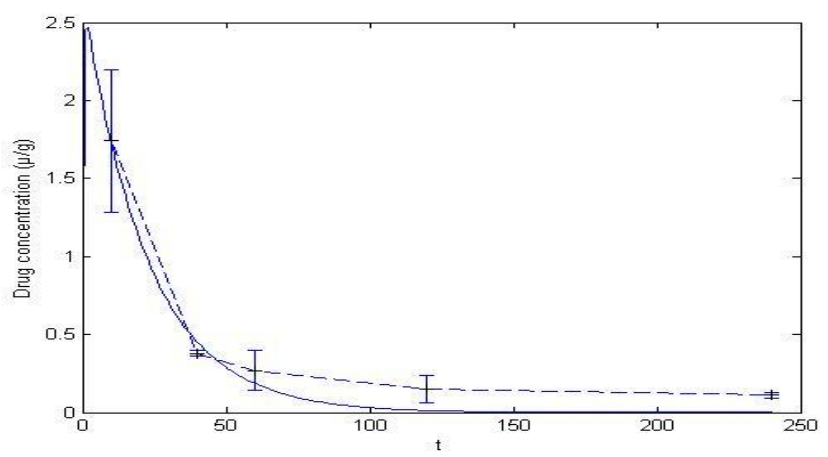

Figure 1. The fitting figure of drug concentration data in the liver tissues

Using Laplace transforming function $c(t)$, we can obtain the transfer function of fitting function, the Fig. (2) is the run results :

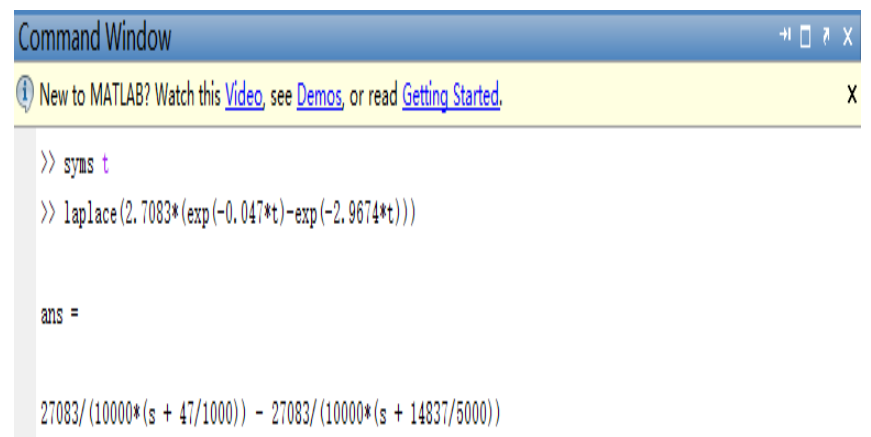

Figure 2. Output the transfer function

The transfer function can be obtained as follows formula (6):

$$
F(S)=\frac{7.9115}{S^{2}+3.0121 S+0.1326}
$$




\section{Sliding Mode Control based on High-gain Observer Design \\ High-gain Observer Design}

The basic ideas of the high-gain observer are as follows: when the high gain observer and nonlinear function satisfy the conditions of a certain relationship, it can replaces the status feedback with the observations, and guarantees the stability of the control system.

It shows that when the gain of high gain observer is large enough, the output feedback controller can reappear the performance of the state feedback controller.

Reference [10] and [15] show that the transfer function obtained by fitting model meets the following formula (7):

$$
\left\{\begin{array}{l}
\dot{\hat{x}}_{1}=\hat{x}_{2}+h_{1}\left(y-\hat{x}_{1}\right) \\
\dot{\hat{x}}=\hat{\phi}(\hat{x}, u, \omega)+h_{2}\left(y-\hat{x}_{1}\right)
\end{array}\right.
$$

In formula (7), $\hat{\phi}(\hat{x}, \mathrm{u}, \omega)$ is the nominal mode of $\phi(x, u, \varpi, d)$.

Consider object in formula (8):

$$
G(s)=\frac{k}{s^{2}+a s+b}
$$

The formula (8) can be expressed as follows formula(9):

$$
\ddot{\theta}=-a \dot{\theta}-b \theta+U(t)
$$

In formula (9), $U(t)=k u(t), \theta(t)$ is a concentration signal, $u$ is Control input. Considering only precise target $F(s)$, we design observer as follows formula(10):

$$
\left\{\begin{array}{l}
\dot{\hat{x}}_{1}=\hat{x}_{2}+\frac{a_{1}}{\varepsilon}\left(y-\hat{x}_{1}\right) \\
\dot{\hat{x}}_{2}=-3.0121-0.1326 \hat{x}_{1}+U+\frac{a_{2}}{\varepsilon^{2}}\left(y-\hat{x}_{1}\right)
\end{array}\right.
$$

\section{Sliding Mode Controller Design}

The tracking error is defined as follows formula (11):

$$
e=\theta_{d}-\theta
$$

In formula (11), $\theta_{d}$ is the given concentration signal, we design the Sliding function as follows formula(12):

$$
s=10 e+\dot{e}
$$


We take sliding mode control law as follows formula (13):

$$
\mathrm{u}(t)=\frac{1}{7.9115}\left(\ddot{\theta}_{d}+3.0121 \hat{\dot{\theta}}+0.1326 \hat{\theta}+10 \hat{s}+1.5 \dot{\hat{e}}\right)
$$

In formula (13), $\hat{\mathrm{e}}=\theta_{\mathrm{d}}-\hat{\theta}, \hat{s}=10 \hat{e}+\hat{\dot{e}}$.

The program of the sliding mode control system that designed in MATLAB / simlink is showed in Fig. (3):

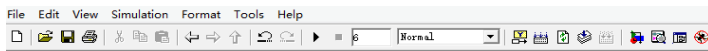

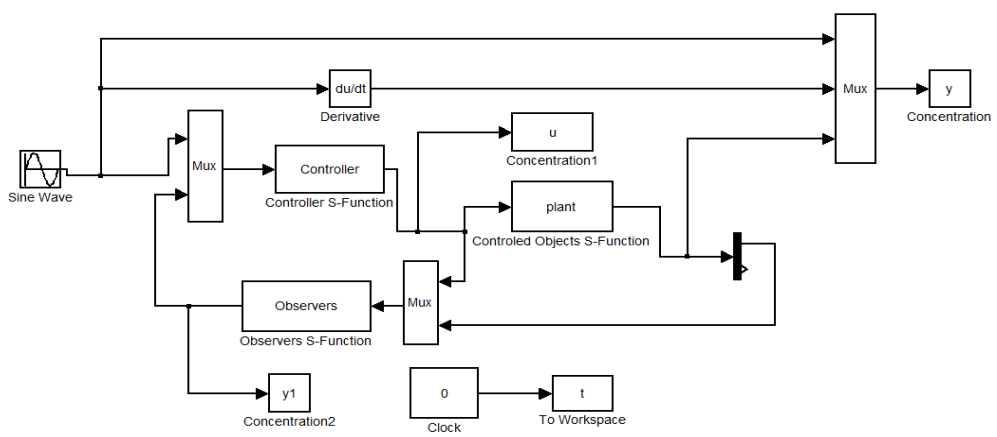

Figure 3. MATLAB / Simulink block diagram

We take $\mathrm{x} 1(0)=0.05, \mathrm{x} 2(0)=0$, as the initial states of the controlled objects, $\theta \mathrm{d}=\sin (\mathrm{t})$ as the concentration instruction, $\varepsilon=0.10, \alpha 1=\alpha 2=1$, as the observer parameters. Running the program, we can get the control effects of the designed control program shown in Fig. 4.
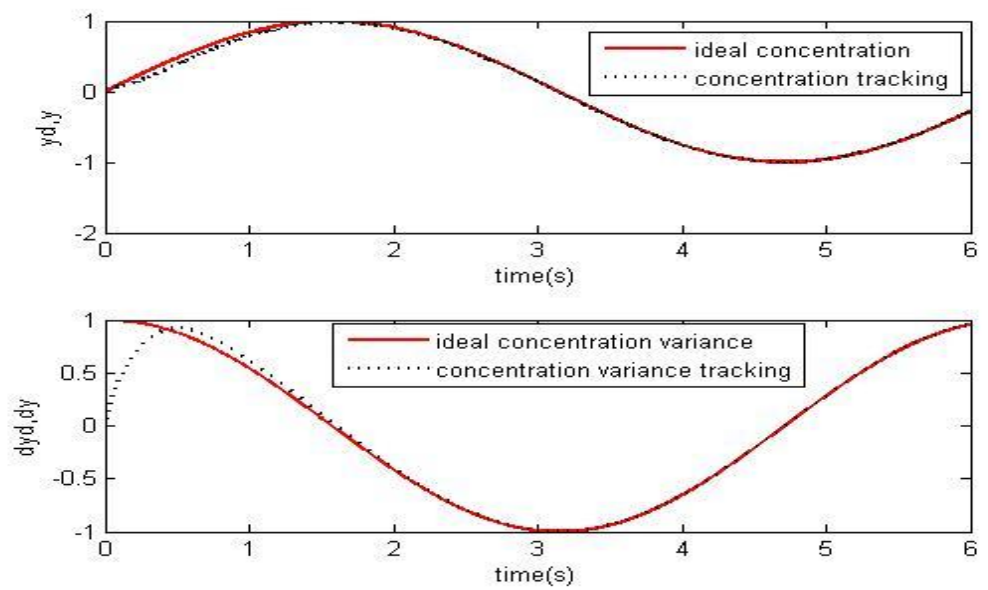

Figure 4. Sliding mode control of concentration and concentration variance tracking

As can be seen from the Fig. 4, by using high-gain observer, without measuring the concentration variance signal, we can get a good tracking performance. Diagram of the entire system is shown in Fig. 5: 


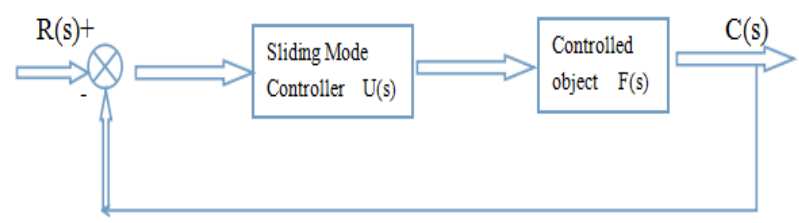

Figure 5 System control diagram

What we can get from the control diagram is the following formula (14):

$$
\frac{C(s)}{R(s)}=\frac{U(s) F(s)}{1+U(s) F(s)}
$$

In formula (14), $C(s)$ is the output function of the entire system, $R(s)$ is the input function of the entire system, in the case of known the output and the total transfer function, by the formula (13), we can obtain the system input function as follows formula(15):

$$
R(s)=\frac{C(s)(1+U(s) F(s))}{U(s) F(s)}
$$

\section{Conclusion}

1). The simulation results show that Sliding Mode Control based on high-gain observer can effectively compensate for the adverse impact on the system that is caused by the outside interference and controls Polydatin concentration in SD rats' liver tissues.

2). The simulation results indicates that the designed observer has a better robustness to changes in measurement parameters and outside interference, and achieves a good tracking effect. The method doesn't need to measure the speed signal, and it has greatly reduced the jitter of the control amount.

3). Data fitting and control have achieved the desired effects, it expresses that the fitting method and control method are applicable to this drug.

4). The mathematical model is clearly and accurately, from which we can easily get the input function and achieve the goal to control the drug concentration in SD rats' liver.

5). The method is also applicable to similar situations, we can estimate the results of tests carried out, the method not only saving a lot of SD rats and equipment, but also saving time for the development of new drugs.

\section{Acknowledgments}

This work was supported by National Natural Science Foundation of China under Grant 81302841 and University Outstanding Talent Support Plan Foundation of Liaoning Province under Grant LJQ2014086. 


\section{Reference}

[1] Huitin Fan, Shilan Ding, Hongsheng Lin. The development of Pharmacological studies on traditional Chinese medicine Polygonum[J]. Traditional Chinese Medicine, 2013: 38 (15): 651-653.

[2] Xiaohua Kong, Lingzhi Zhou. Study Progress of Giant Knotweed Rhizome[J]. Traditional Chinese Medicine, 2009, 15(5): 107-110.

[3] Meihong Guo, Xiaodan Huang, Ying Fan, etc. The effects of Huzhangjiangzhi granules on hyperlipidemia in rats[J].Chinese New Clinical Medicine. 2015, 8(10): 11-16.

[4] Yuegang Wang, Chunhua Jin, Ping-sheng Wu. Effect of polydatin on protein kinase $\mathrm{C}$ activity of myocardial cells in ischemia and hypoxia[J]. Chinese Pharmacological Bulletin, 2007, 5 (5): 590-593.

[5] Salvatore De Maria, Llaria Scognamiglio, AngelaLombardi. Polydatin, A natural precursor of resveratrol, induces cell cycle arrest and differentiation of human colorectal Caco-2 cell[J]. Journal of Translational Medicine. December 2013, 11: 264.

[6] KS Zhao, C Jin, X Huang, J Liu. The mechanism of Polydatin in shock treatment[J]. Clinical Hemorheology \& Microcirculation, 2003, 29(3-4): 211-217.

[7] Shouhong Gao. Studies on Biopharmaceutical Analysis and Preclinical Pharmacokinetics of Polydatin. Shanghai: master's degree thesis of Second Military Medical University of PLA. 2005.

[8] Weibing Gao .Variable structure control theory and design. Beijing:Science Press, 1996.

[9] Xiaojiang Mu, Yangdan Chen. Summary of variable structure theory control[J]. Control Engineering, 2007, 14(S2): 5-9.

[10] Jinkun Liu. Sliding Mode Control MATLAB simulation. Beijing: Tsinghua University Press, 2015: 278-285.

[11] Giordano F R, Weir M D, Fox W P. A first course in mathematical modeling. 3th, ed. [S.L.]: Brooks/Cole, 2003.

[12] Ming Han. Mathematical modeling case. Shanghai: Tongji University Press, 2012.6.

[13] Shulan Zhao. MATLAB modeling and simulation. Beijing: Tsinghua University Press, 2013.

[14] Chaoying He, Shaoyu Wang. MATLAB application and Experimental Guide. Beijing, Electronic Industry Press, 2013: 100-125.

[15] H. K. Khalil. High-Gain Observers in Nonlinear Feedback Control [C]. International Conference on Control Automation and Systems, Seoul: 2008: 249-269. 